
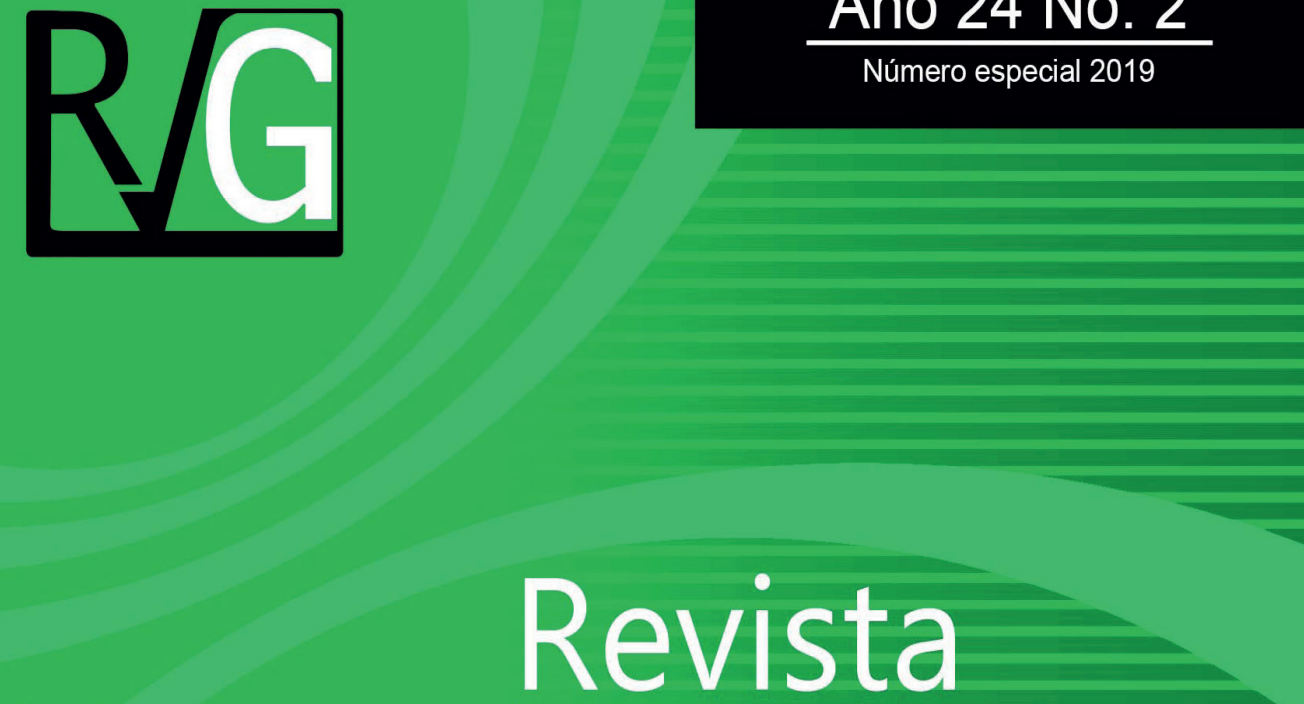

Venezolana de

$\frac{1}{6}$

$\frac{\mathbb{O}}{\frac{1}{0}}$
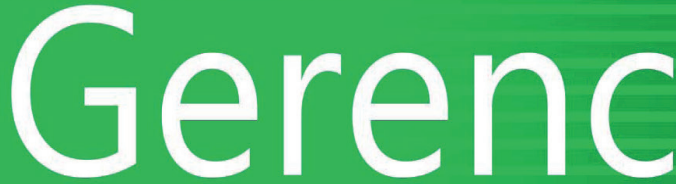

0

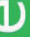

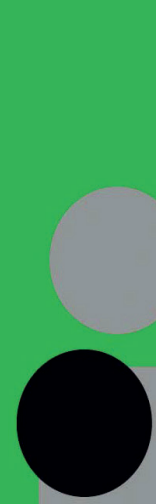

ro

u

$>$

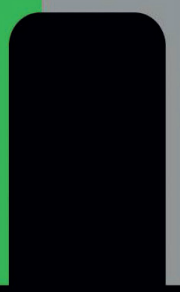




\title{
Tiendas Hard Discount y su incidencia en los minimercados boyacenses
}

\author{
Molina Valencia, Ricardo Gustavo ${ }^{1}$ \\ González Millán, Oscar Ulises ${ }^{2}$ \\ González Millán, José Javier ${ }^{3}$
}

\section{Resumen}

Por estos días se evidencia la llegada a todo Colombia de los almacenes denominados Hard Discount (de descuento duro), que han cautivado a sus clientes con la estrategia de bajos precios en sus productos, razón por la cual han venido invadiendo masivamente el comercio en el departamento de Boyacá. Por lo antes expuesto, el objetivo de esta investigación fue conocer las implicaciones que este modelo de negocio tiene para los minimercados existentes en las tres principales ciudades del departamento de Boyacá (Tunja, Duitama y Sogamoso). En la parte metodológica la investigación fue de corte descriptivo explicativo, utilizando como instrumento de recolección la encuesta semiestructurada, dirigida a los propietarios minimercados, la cual fue aplicada a 400 unidades muestrales. Como resultado se evidencio que los factores que han afectado a los tenderos tienen que ver directamente con la comercialización de los productos a un bajo precio, la carencia de estrategias de marketing, lo que paulatinamente ha ido disminuyendo la cantidad de clientes y por ende las ventas diarias se han reducido entre 1 USD y 3 USD diarios.

Palabras Clave: descuento duro; precios; consumidores; minimercados.

Recibido: 11-09-19 Aceptado: 10-11-19

Magister en dirección estratégica de la UCl México, Profesor/Investigador Escuela de Administración de Empresas, Facultad Sede Sogamoso, Universidad Pedagógica y Tecnológica de Colombia, Sogamoso Coordinador grupo Investigación MANAGEMENT, ricardo.molina@uptc.edu.co , https://orcid.org/0000-00022936-9042

2 Magister en Salud y Seguridad en el trabajo de la Universidad Nacional de Colombia, Profesor/Investigador Escuela de Ingeniería de Minas, Facultad Sede Sogamoso, Universidad Pedagógica y Tecnológica de Colombia, Sogamoso, Investigador grupo GEAM, oscar.gonzalez02@uptc.edu.co, https://orcid.org/00000002-2943-5492

3 Doctor (c) en Administración Universidad Autónoma de Querétaro, Profesor/Investigador Escuela de Administración de Empresas, Facultad Sede Sogamoso, Universidad Pedagógica y Tecnológica de Colombia, Sogamoso, Investigador grupo Management, javier.gonzalezmillan@uptc.edu.co, https://orcid. org/0000-0002-6233-1804 


\title{
Hard Discount Stores and their impact on the mini-markets of Boyacenses
}

\begin{abstract}
These days it is evident the arrival throughout Colombia of the so-called Hard Discount stores, which have captivated their customers with the strategy of low prices on their products, which is why they have been massively invading trade in the Boyacá department. Therefore, the objective of this research was to know the implications that this business model has for the minimarkets in the three main cities of the department of Boyacá (Tunja, Duitama and Sogamoso). In the methodological part, the investigation was descriptive descriptive, using the semi-structured survey as a collection instrument, aimed at minimarkets owners, which was applied to 400 sample units. As a result, it was evident that the factors that have affected the shopkeepers have to do directly with the commercialization of the products at a low price, the lack of marketing strategies, which gradually has been decreasing the amount of customers and therefore the sales daily have been reduced between 1 USD and 3 USD daily.
\end{abstract}

Keywords: hard discount; prices; consumers; minimarkets

\section{Introducción}

La situación económica de población colombiana aunada al bajo poder adquisitivo de los habitantes del país (ANDI,2017) ha venido generando estrategias de subvención financiera para poder apalancar la estabilidad económica y el mantenimiento de una canasta familiar asequible a los diversos estratos económicos, es por tanto, que ha llegado a Colombia el denominado modelo Hard Discount (HD) o almacenes de descuento duro, en donde su estrategia comercial de precios bajos, marcas propias y diversidad de productos ha inundado el mercado colombiano, por lo cual, el departamento de Boyacá no ha sido la excepción en la implantación de éste esquema, que se ha hecho presente por medio de tres grandes superficies ubicadas estratégicamente en las principales ciudades (Cámara de Comercio de Tunja, 2017).

Las tiendas de descuento duro, han sido definidas por un directivo de la cadena D1 (Semana, 3/4/2017:4) como "el negocio cuyo propósito es establecerse en los barrios para tener mayor cercanía con la clientela, mantener precios competitivos y tener buen abastecimiento de los productos básicos que demandan los consumidores", así pues, se puede considerar que el modelo de negocio además de tener precios cómodos, busca ubicarse en diferentes barrios para establecer buenas relaciones con sus clientes (Schwalb \& García, 2019). Aunque el efecto y el servicio es indiscutible, el fenómeno ha venido teniendo consecuencias sobre los minimercados existentes en el departamento, pues el abastecimiento de éstos se ha hecho a través de marcas 
tradicionales de las grandes superficies, con precios diferenciados y con muy pocas estrategias promocionales (Martín, 2001), dicho fenómeno constituye un efecto positivo para los compradores más no en esa misma medida para los minimercados, es por esto que, resulta pertinente conocer el las implicaciones e incidencia del modelo HD sobre los minimercados del departamento (González, Cerón \& Alcázar, 2010). En lo concerniente a la metodología, la investigación se configuró con base en el tipo descriptivo- explicativo, aplicando 400 encuestas semiestructuradas a los propietarios de los minimercados de las tres principales ciudades (Tunja, Duitama y Sogamoso) del departamento de Boyacá, con relación al tratamiento de la información, se acudió al modelamiento estadístico a través del paquete SPSS Vs 24, tomando un diseño estratificado por grupos ciudades a fin de hacer el análisis descriptivo-explicativo.

\section{Marco Teórico Hard Discount Stores (HDS)}

Para el caso colombiano existen actualmente tres superficies de Descuento Duro reconocidos por ofrecer productos a bajo precio (Gómez, Jiménez, \& Perdiguero, 2015), dichos almacenes trabajan bajo la estrategia que otras superficies a nivel mundial han desarrollado. En relación al origen

Las tiendas de descuento duro fueron inventadas en 1948 por los hermanos Karl y Teo Albrecht, que desarrollaron Aldi en Alemania. Estas se caracterizan por tener un surtido limitado, política constante de bajos precios, control sistemático de costos y oferta de marcas propias (Dinero, 2/4/2016: 5).

En Colombia, la creación de los primeros supermercados proviene de un joven español quien vio en este país la oportunidad de emprender un negocio innovador y de grandes beneficios económicos, en la historia se relata que,

El primer gran creador del formato de supermercado en el país fue don José Carulla Vidal, quien brindó a sus clientes calidad en los productos que vendía en el Escudo Catalán, nombre con el que bautizó originariamente su negocio. En corto tiempo se convirtió en el lugar favorito de familias bogotanas para las cuales la novedad del negocio y del servicio al cliente resultaron tan atractivos que cuatro años más tarde "El Escudo Catalán" no daba abasto y su propietario tuvo que abrir una sucursal, que posteriormente se convirtió en supermercados Carulla. (Historia de supermercados Carulla citada por El tiempo, 2005:1)

En esta sincronía, Torres (2012:115), sugieren que los supermercados son los que comprenden a "Megamercados, líneas completas de productos desde alimentos, ropa, productos electrónicos, refacciones para coches, productos para mantenimiento de casas (...) Hiper-mercados, líneas completas de productos y los alimentos", consecuentemente con lo anterior, y según la Asociación Paulista de Supermercado, el sector de supermercados, por su representación en el escenario nacional y mundial, juega un papel importante en el movimiento de la sustentabilidad, educación ambiental y concienciación (Martínez, Silveira, Braga, \& Da Silva, 2017:153). Así mismo, autores como Lourenço \& Gijsbrechts (2013: 369) indican que "el impacto en el HD está en la buena relación calidad-precio-posicionamiento, como ingrediente clave de su éxito", dada esta consideración, el efecto en 
el poder adquisitivo del consumidor evoca productos económicos de poco reconocimiento, pero que se constituyen en consumos básicos dentro de la canasta familiar de los consumidores que a la postre se convierten en clientes (González, 2009).

\subsection{Definición de almacenes Hard Discount Store (HDS)}

Vroegrijk, Gijsbrechts \& Campo (2016:300) ponen en consideración que los "HDS han conquistado los corazones y mentes de un segmento creciente de consumidores, que creen que proporcionan un mejor valor de dinero que los formatos tradicionales", en otras palabras, las tiendas Hard Discount se han convertido en un formato de almacenes que han ganado clientes bajo la premisa de poder adquirir más productos a menor precio. De otro lado la definición de tiendas de descuento duro se da como una tienda de ahorro teniendo en cuenta que:

La compra de todos los productos a un Hard Discount en lugar de un Traditional Store (TS), puede resultar en un ahorro sustancial por dos razones. En primer lugar, hacer compras en un HD permite a los consumidores a adquirir artículos comestibles a precios mucho más bajos, pues las tiendas de alta definición son capaces de ofrecer productos de alta calidad a precios que están muy por debajo de los precios regulares de las principales marcas nacionales; En segundo lugar, hacer compras en un HD puede ejercer un impacto adicional en el gasto cuando no sólo afecta el precio por unidad de compra, sino también el número de productos que se compran. (Gijsbrechts, Campo \& Vroegrijk, 2018:4)
En concordancia con lo anterior, Prada \& Ocampo (2016:78) sugieren que "Este innovador modelo está diseñado para minimizar el costo al cliente representado en reducciones en logística, personal, transporte, publicidad y decoración. La idea se basa en trasladar los ahorros en las áreas mencionadas y llevarlos a los precios finales de los productos", como se puede observar el Hard Discount lleva tiempo desarrollándose en el mercado esto ha permitido estudiar el impacto que estos causan, en este sentido Igami citado por Zipitría (2011:78) demuestra "que el ingreso de almacenes HD aumenta la probabilidad de que otros grandes supermercados salgan del mercado", es decir que como consecuencia de los bajos precios muchos supermercados han tenido que cerrar sus puertas al no contar con estrategias que les permitan igualar precios con los mencionados almacenes. (Salhin, 2013)

\subsection{Características de los Establecimientos Hard Discount}

La característica más notoria de las tiendas de descuento duro son los bajos precios que manejan (López \& Peñalosa, 2019), pero conviene conocer las características internas de estos almacenes, para lo cual Gijsbrechts, Campo \& Vroegrijk (2018:170), reconoce las Siguientes: (cuadro 1).

Adicionalmente otros autores (Gijsbrechts, Campo \& Vroegrijk, 2018:175) destacan que,

La estrategia de la agrupación, que se utiliza con frecuencia por las cadenas de descuento duro, significa que varios medios se abren simultáneamente cerca de los almacenes logísticos, debido a que 


\section{Cuadro 1 \\ Características Supermercados Hard Discount}

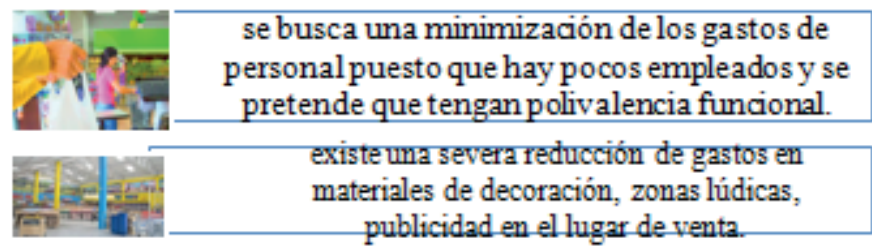

DIf $\begin{gathered}\text { no existe asistencia o servicio a los clientes sobre } \\ \text { los productos ofertados e, incluso, por las bolsas } \\ \text { deplástico hay que pagarunprecio. }\end{gathered}$

serealizan grandes volúmenes de compra a los
proveedores para conseguir precios ajustados

Fuente: Elaboración propia a partir de Gijsbrechts, Campo \& Vroegrijk (2018:170)

uno de los factores clave del éxito de una red de descuento se basa en el control de costes. Permite reducir la publicidad y los costes logísticos y para bloquear los mercados antes que los competidores. (Cliquet \& Guillo, 2013).

\subsection{Clasificación de las tiendas de descuento}

Dentro de la clasificación de tiendas de descuento se encuentran las siguientes categorizaciones:

- Descuento duro - clásico: De acuerdo con Vicuña citado por Beltrán (2016:13), este tipo de tienda "se sitúa especialmente en los centros urbanos, contiguo a empresas que venden mercancías frescas ofreciendo productos similares y otros con los que logra complementar su surtido. (Supermercado agresivo)", en tal medida, el establecimiento conserva ciertas particularidades del supermercado habitual y las tiendas de descuento duro brindando dos veces o más productos en cuanto a los almacenes HDS.

- Descuento duro - nuevo formato. Al tenor de lo expresado por (Beltrán, 2016:13), dicho establecimiento "suele tener un espacio amplio entre 700 y 900 m2 - y una cantidad de productos mayores, se sitúa en lugares ampliamente lejanas a los centros de la ciudad con el fin de disminuir costos" (Supermercado).

\subsection{Clasificación de las tiendas de mercado}

Las tiendas de mercado son empresas que se han considerado elementales por mucho tiempo, por lo cual los gerentes, administradores y dueños han buscado darle un valor agregado que permite clasificarlos así: (Cabrera y García, citados por Hernández, Portillo, Romero \& Hernández (2015:120)

- Tiendas por su apariencia: un segmento de los clientes evalúa en 
estas tiendas el aspecto físico cuyos rasgos principales son dimensión, organización, e higiene como factores importantes para conservar y fortalecer las conexiones permanentes. Corresponde a un espacio cómodo de acuerdo a Bergadaà \& Del Bucchia, citados por Morales, (2012:2)

- Tiendas por su familiaridad (minimercado): La tienda es vista como un lugar correspondiente a una cultura propia de complicidad. Un lugar en el que los compradores se consideran la tienda como un segundo hogar, inclusive existe cierta confianza. En términos sociales expresados por Guedon citado por Morales (2012:2) son de gran significado en la cotidianidad o cuando aparece una emergencia.

- Tiendas por su rol de intermediación: los establecimientos asumen la tarea de servir de medio logístico entre productores, mayoristas o minoristas y clientes, un grupo de compradores las conciben como mediadores, como el medio tradicional de gran importancia en la vida de la comunidad. (Bergadaà \& Del Bucchia, citados por Morales, 2012).

Bossa (2012), supone que las tiendas minoristas o autoservicios son susceptibles a ser afectadas por los cambios en el mercado como la entrada de competidores con precios menores, puesto que no cuentan con un poder de negociación con los proveedores debido a las pequeñas unidades de mercancía que manejan y aunque los almacenes Hard Discount son un modelo de negocio nuevo en la región tienden a causar un alto impacto en el comercio regional. En apoyo a lo anterior, es pertinente acotar que el concepto de mercado de descuento, se orienta a la clase media y baja, destacando que:

La importancia de la tienda se debe a que en ella se desarrollan prácticas comerciales basadas en la confianza mutua entre tenderos y consumidores; a los factores de cercanía, a la miniaturización de productos expendidos, al fiado confiando sólo en la palabra, al regateo y a la ñapa. (Ramírez \& Pachón, citados por Plazas, 2008:40)

\section{Aproximaciones metodológicas del estudio}

A continuación se detallan algunos aspectos metodológicos relevantes para la investigación.

\subsection{Tipo y método de investigación}

Esta investigación es de tipo descriptivo-explicativo, en la cual se lograron establecer las características y factores influenciadores de las tiendas HD en los minimercados (Méndez, 2006) del departamento de Boyacá. En lo pertinente al método éste correspondió al deductivo, para estar acorde con la explicación de implicaciones sobre el objeto de estudio (Camacho, 2003).

\subsection{Fuente y técnica de recolección de información}

La fuente primaria (Tamayo, 2002) correspondió a los propietarios de los minimercados de las tres ciudades más importantes del departamento de Boyacá (Tunja, Sogamoso y Duitama). Como técnica de recolección de información se trabajó con la encuesta semiestructurada adaptada de Caicedo 
y Quiceno (2015). Con relación a las fuentes de segundo orden fueron otras investigaciones, utilizando como técnica la revisión documental y el análisis de las bases de datos.

\subsection{Población, muestra, tipo de muestreo y tratamiento de la información.}

Se aplicaron 400 encuestas a los propietarios de los minimercados (Tunja=160; Sogamoso= 120 ;
Duitama=120). En lo pertinente al diseño muestral (Sampieri, Fernández y Baptista, 2010) se llevó a cabo el estratificado por el método de conveniencia debido a dificultades con respecto al acceso de la información.

\subsection{Ficha técnica propuesta}

El cuadro 2 presenta la ficha técnica de los elementos relevantes del diseño muestral:

\section{Cuadro 2}

\section{Ficha técnica del muestreo}

\begin{tabular}{ll}
\hline & Ficha técnica \\
\hline Fecha de realización encuesta & Julio - Noviembre de 2019. \\
Institución & UPTC - Sogamoso \\
Facultad & Sede Sogamoso \\
Escuela & Administración de Empresas \\
Departamento & Boyacá \\
Población & Ciudades de Tunja, Duitama y Sogamoso \\
Tamaño de la población & 400 encuestas \\
Procedimiento de recolección & Muestra por conveniencia \\
Sistema de procesamiento & SPSS ${ }^{\text {TM }}$ (v. 24) \\
Metodología & Encuesta. Cuestionario \\
Unidad de análisis & Propietarios de minimercados \\
Realizador del estudio & Investigadores del proyecto \\
Prueba piloto & Se aplicaron un total de 10 encuestas: 5 en \\
\hline
\end{tabular}

Fuente: Elaboración propia

\section{Factores que inciden en los minimercados}

En la presente investigación se buscó identificar los factores relevantes que inciden en los minimercados de las ciudades de Tunja, Duitama y Sogamoso y la forma en que han afectado o beneficiado las tiendas de descuento duro a los pequeños comerciantes de las diferentes ciudades. En este sentido, se indago sobre variables como la escolaridad del tendero (Tabla 1), los precios, la calidad de los productos, la variedad, la atención al cliente y las promociones, encontrando que: 


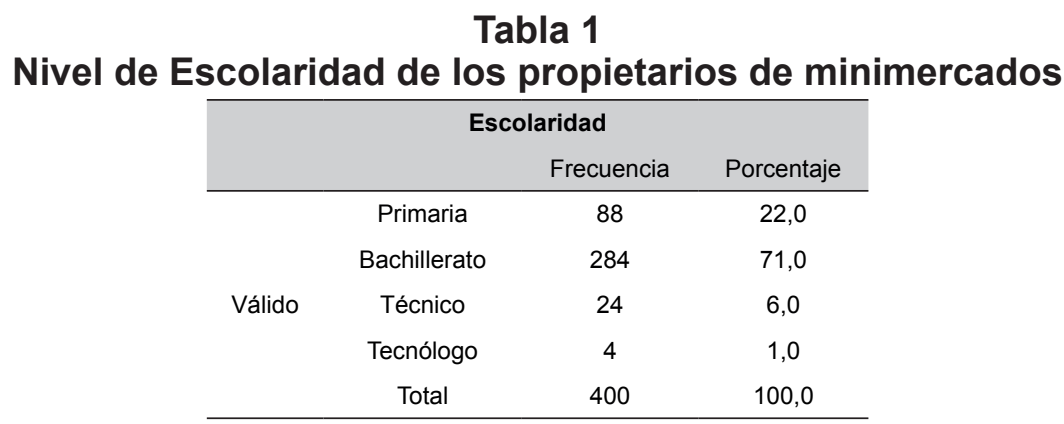

Fuente: Elaboración propia

Sobre el nivel de escolaridad de los tenderos corresponden en su mayoría al bachillerato $(71 \%)$ y primaria $(22 \%)$, llama la atención que un pequeño porcentaje de ellos tienen estudio como técnico o tecnólogo, por lo cual, se evidencia que algunos de ellos no tienen el conocimiento para aplicar técnicas de mercadeo que les permitan aprovechar las oportunidades del mercado y enfrentar los cambios en el mercado. En segundo lugar, se indagó sobre la antigüedad del negocio frente a la escolaridad del tendero (Tabla 2), se observa que las tiendas con mayor tiempo en el mercado corresponden entre 11 y 20 años y son administradas por personas bachilleres y con básica primaria; seguidas de tiendas entre 6 y 10 años de antigüedad con las mismas características de sus dirigentes.

Tabla 2

Nivel de Escolaridad vs tiempo del minimercado

\begin{tabular}{|c|c|c|c|c|c|c|}
\hline \multicolumn{7}{|c|}{ Tabla cruzada Escolaridad ¿ ¿Hace cuánto tiempo tiene su negocio? } \\
\hline \multirow{2}{*}{\multicolumn{2}{|c|}{ Entre 1 y 5 años }} & \multicolumn{4}{|c|}{ ¿Hace cuánto tiempo tiene su negocio? } & \multirow[b]{2}{*}{ Total } \\
\hline & & $\begin{array}{c}\text { Entre } 6 \text { y } \\
10 \text { años }\end{array}$ & $\begin{array}{c}\text { Entre } 11 \text { y } \\
20 \text { años }\end{array}$ & $\begin{array}{l}\text { Entre } 21 \text { y } \\
30 \text { años }\end{array}$ & & \\
\hline \multirow{4}{*}{ Escolaridad } & Primaria & 4 & 20 & 48 & 16 & 88 \\
\hline & Bachillerato & 28 & 56 & 144 & 56 & 284 \\
\hline & Técnico & 0 & 16 & 8 & 0 & 24 \\
\hline & Tecnólogo & 4 & 0 & 0 & 0 & 4 \\
\hline \multicolumn{2}{|c|}{ Total } & 36 & 92 & 200 & 72 & 400 \\
\hline
\end{tabular}

Fuente: Elaboración propia

Se logra establecer que los tenderos conocen por medio de la experiencia el manejo de sus negocios, el comportamiento de sus clientes, la negociación con los proveedores y las necesidades específicas de sus compradores; en su mayoría son tiendas que fidelizan sus clientes por el crédito 
que les otorgan durante el mes. Es importante indicar que los autoservicios son una forma de obtener ingresos para muchas personas que, por factores como la edad, la escolaridad, la familia o la raza, laboralmente no tienen muchas oportunidades, pero encuentran en el comercio al por menor una forma de subsistir y en la mayoría de las ocasiones no se busca capacitación para el crecimiento empresarial. El tercer elemento analizado, se orientó a indilgar sobre los indicadores de disminución de clientes y el número de clientes promedio del establecimiento (Tabla 3), ante lo cual la investigación reveló que el número de clientes diarios es de 21 a 50 y del total de encuestados 284 han notado que los clientes han disminuido, la razón la aducen a la llegada de las tiendas de descuento duro, las ventas por catálogo, los vendedores informales y la llegada de comerciantes extranjeros.

\section{Tabla 3}

\section{Clientes Promedio vs Disminución de Clientes del minimercado}

\begin{tabular}{ccccc}
\hline $\begin{array}{c}\text { Tabla cruzada ¿Cuál es el número de clientes promedio que compran en su tienda en el transcurso } \\
\text { del día? * ¿Ha percibido alguna disminución en la cantidad de clientes por día en el último año? } \\
\text { ¿Ha percibido alguna disminución en } \\
\text { Si }\end{array}$ & $\begin{array}{c}\text { ¿Ha cantidad de clientes por día en el } \\
\text { último año? }\end{array}$ & Total \\
& & No & 0 & 72 \\
\hline $\begin{array}{c}\text { ¿Cuál es el número de clientes } \\
\text { promedio que compran en su } \\
\text { tienda en el transcurso del día? }\end{array}$ & De 21 a 50 & 284 & 4 & 288 \\
& De 51 a 100 & 32 & 0 & 32 \\
Total & Más de 100 & 8 & 0 & 8 \\
\hline
\end{tabular}

Fuente: Elaboración propia

Todos estos factores han llevado al cierre de muchas tiendas que han sido tradicionales en las diferentes ciudades y que no han podido responder a estas dificultades.

El siguiente factor intento identificar de manera cruzada la relación entre la disminución de los clientes y la disminución de las compras promedio que al día afectan al establecimiento
(Tabla 4), dando cuenta que los factores que más afectan el normal desarrollo de su actividad tienen en común la comercialización de los productos a un bajo precio específicamente las tiendas de descuento duro con las promociones y ganchos de mercado con sus marcas propias, factor que poco a poco ha ido disminuyendo sus ventas diarias. 


\section{Tabla 4 \\ Disminución de Clientes vs Disminución de compras en minimercados}

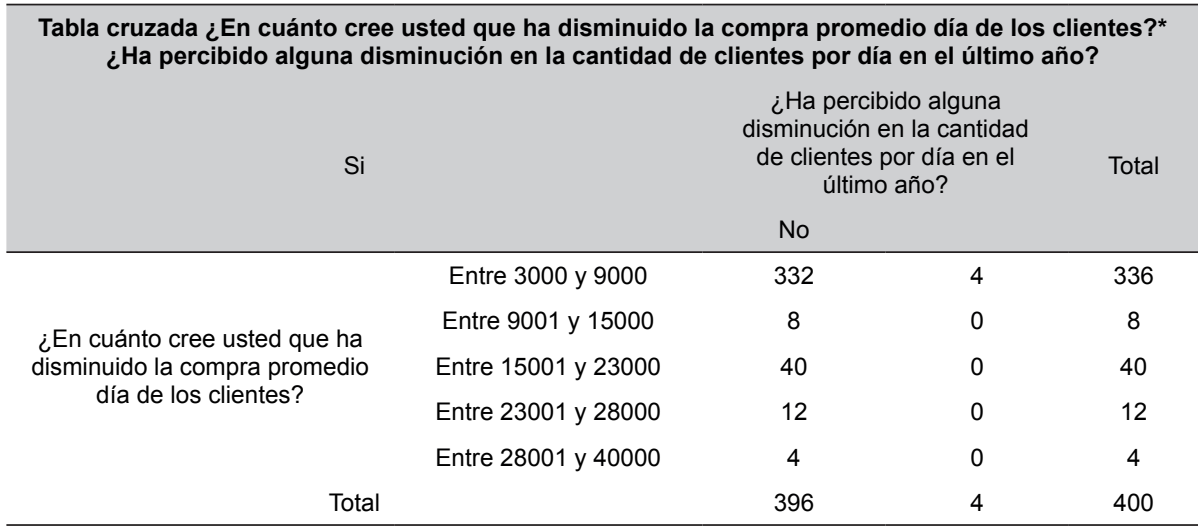

Fuente: Elaboración propia

Por tanto, quienes han observado la disminución en sus clientes (332 tenderos) indican que cada uno han dejado de comprar en promedio entre 3000 y 9000 pesos y 40 tenderos que las ventas se han reducido entre 15001 y 23000 pesos diarios por cliente, estas cifras llaman la atención dado que estos negocios dependen de sus ventas para cumplir con gastos básicos como arriendo del local, servicios, proveedores, entre otros.

El quinto elemento investigado se refirió a escudriñar que otros factores se le pueden atribuir a la disminución en las ventas de los pequeños tenderos, dentro de los cuales subyacen la diferencia en los precios frente a la competencia, en tal sentido, 356 de ellos indican que procuran igualar los precios con los supermercados y nuevas tiendas con el propósito de mantenerse en el mercado, aunque esto implique disminuir el margen de utilidad o comprar en grandes cantidades para aprovechar descuentos por parte de los proveedores. Una sexta variable se orientó a averiguar si el establecimiento posee estrategias de venta y sistemas promocionales (Tabla 5), a lo cual los propietarios manejan promociones, en consecuencia la mayoría de ellos no tienen estrategias (392) y 240 de ellos no realizan promociones de ningún tipo en sus negocios, este resultado se puede asociar a la escolaridad de los tenderos que como se había mencionado en su mayoría solo poseen primaria y bachillerato por ende sus conocimientos en mercadeo y publicidad son mínimas. 


\section{Tabla 5 \\ Estrategias de Ventas vs promociones en minimercados}

\begin{tabular}{ccccc}
\hline \multicolumn{2}{c}{ Tabla cruzada ¿Maneja promociones en su tienda?* ¿Tiene estrategias de venta en su tienda? } \\
¿Tiene estrategias de venta en su \\
tienda?
\end{tabular}

Fuente: Elaboración propia

También puede influenciar en este punto, la cultura empresarial de la región (González, Rodríguez \& Moreno, 2014)., que apunta a invertir lo menos posible en actividades de Marketing, adicionalmente a esto, es importante resaltar que en algunas ciudades las cámaras de comercio y el Servicio Nacional de Aprendizaje (SENA) realizan diversas capacitaciones para pequeños comerciantes, pero ante los resultados al parecer no son recibidas o no se aplican luego de terminado el curso. El séptimo ítem pretendió medir el grado de afectación por el surgimiento de las tiendas de descuento duro en relación con el tiempo de surgimiento del negocio (Tabla 6) en escala de 1-10, ante lo cual, la llegada de las tiendas de descuento duro es calificada en 8 por 336 de ellos, este corresponde a un alto grado, también se evidencia que los negocios más afectados son los más antiguos, es decir, los que llevan en el mercado más de 6 años.

\section{Tabla 6}

\section{Grado de afectación por las HD vs tiempo en el negocio}

\begin{tabular}{|c|c|c|c|c|c|c|}
\hline \multicolumn{7}{|c|}{$\begin{array}{l}\text { Tabla cruzada Marque de } 1 \text { a } 10 \text {, el grado de afectación que ha tenido su tienda debido a la aparición de las nue- } \\
\text { vas superficies, siendo } 1 \text { (no afectado) y } 10 \text { (muy afectado) ¿Hace cuánto tiempo tiene su negocio? }\end{array}$} \\
\hline \multirow[b]{2}{*}{ Entre 1 y 5 años } & \multicolumn{6}{|c|}{ ¿Hace cuánto tiempo tiene su negocio? } \\
\hline & & $\begin{array}{l}\text { Entre } 6 \text { y } \\
10 \text { años }\end{array}$ & $\begin{array}{l}\text { Entre } 11 \text { y } \\
20 \text { años }\end{array}$ & $\begin{array}{c}\text { Entre } 21 \text { y } \\
30 \text { años }\end{array}$ & & Total \\
\hline \multirow{8}{*}{$\begin{array}{l}\text { Marque de } 1 \text { a } 10 \text {, el grado de } \\
\text { afectación que ha tenido su tienda } \\
\text { debido a la aparición de las nuevas } \\
\text { superficies, siendo } 1 \text { (no afectado) y } 10 \\
\text { (muy afectado) }\end{array}$} & 2 & 0 & 0 & 4 & 0 & 4 \\
\hline & 3 & 0 & 4 & 4 & 0 & 8 \\
\hline & 4 & 0 & 0 & 4 & 0 & 4 \\
\hline & 5 & 0 & 4 & 0 & 0 & 4 \\
\hline & 6 & 0 & 4 & 0 & 0 & 4 \\
\hline & 8 & 8 & 76 & 184 & 68 & 336 \\
\hline & 9 & 0 & 0 & 0 & 4 & 4 \\
\hline & 10 & 28 & 4 & 4 & 0 & 36 \\
\hline Total & & 36 & 92 & 200 & 72 & 400 \\
\hline
\end{tabular}

Fuente: Elaboración propia 
Este resultado se da principalmente por la falta de conocimiento y aplicación de estrategias de planeación en áreas de mercadeo, manejo de inventarios, contabilidad, entre otros. De la misma manera, la tabla 7 pretendió auscultar el manejo de servicio al cliente por medio del servicio telefónico para atender domicilios y pedidos de los clientes y en atención a esto cuantas llamadas recibe en promedio al día.

\section{Tabla 7}

\section{Nivel de llamadas vs atención telefónica para pedidos de clientes}

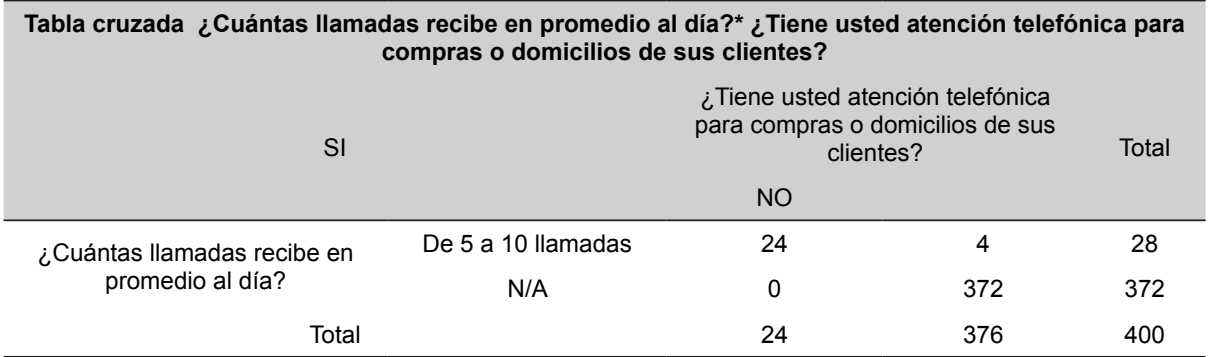

Fuente: Elaboración propia

En esta medida, la encuesta arrojo que 376 tiendas No realizan ventas por este medio, tan solo 24 negocios venden a través de llamadas telefónicas y reciben de 5 a 10 llamadas diarias. La forma común de venta en estos negocios corresponde a la venta directa ya que según indican los tenderos los clientes valoran el servicio personalizado y prefieren seleccionar ellos mismos sus productos y precios, por tanto, es mayor la satisfacción de los clientes. En este mismo punto se indago sobre los factores que el propietario consideraba más afectaban su negocio en una escala 1 a 5 siendo 1 la variable que más afecta a los tenderos de las ciudades, la cual corresponde a los precios, aunque traten de igualar los precios sigue siendo esta la variable que más afecta junto con las promociones (grado 2) ya que las tiendas de descuento duro aproximadamente cada quince días realizan descuentos del 10, 20 y $30 \%$ en toda la tienda. En tercer lugar afecta la atención al público ya que en dichos lugares existen personas especialistas en ventas y asesoran al cliente en cuanto a la variedad de precios y productos. El factor que afecta en menor medida es la amplitud de horarios teniendo en cuenta que normalmente las tiendas de barrio están abiertas aproximadamente desde las 6.30 am hasta las 9 pm mientras que los almacenes como Ara, D1, justo y bueno inician la atención al público hacia las 9 am y cierran hacia las 8.30 pm.

\section{Implicaciones de la estrategia de descuentos duros}

La investigación se confronta y corrobora lo expuesto por Beltrán (2016) el cual concluye en su investigación que, las tiendas de barrio difícilmente 
desaparecerán, pero efectivamente han venido perdido consumidores, y aunque son parte fundamental de la cadena de suministro de alimentos en Colombia, el $71 \%$ de los encuestados manifestó que los clientes han disminuido debido a la llegada de las tiendas de descuento duro y que este factor ha llevado al cierre de muchas tiendas tradicionales en las diferentes ciudades. En tal medida, el futuro de las tiendas y minimercados tiende a ir desapareciendo de manera progresiva ya que a los tenderos les es difícil cumplir con sus obligaciones financieras y administrativas como lo hacían antes de la llegada de los Hard Discount.

De otro lado, la investigación desarrollada por Jaramillo, Neita, Pirazán \& Villarraga, (2017) menciona que, aunque estos almacenes venden a precios bajos, no significa que los productos sean los más baratos teniendo en cuenta que los gramajes y pesos son menores que los ofertados en las tiendas tradicionales, por tanto, enfatizan que este aspecto no ha sido detectado por los clientes, los cuales se inclinan por la economía que por las calidades en las compras. En acuerdo a lo antes expuesto la presente investigación coincide en que los factores que han afectado el normal desarrollo de la actividad en las tiendas de barrio tienen en común la comercialización de productos a un bajo precio específicamente las tiendas de descuento duro con promociones quincenales y semanales.

Almeyda (2016) mediante el estudio El fenómeno de las tiendas de descuento en el mercado del retail colombiano Señala que el tema de los precios es un factor diferenciador a corto plazo entre los diversos formatos de supermercados, ya que en algún momento la economía en Colombia logrará estabilizarse, por tanto, factores como la calidad y la facilidad (ubicación y disponibilidad) para la compra de un artículo, cobraran más importancia que el mismo precio del producto. En contraposición en este estudio se observó que los tenderos procuran igualar los precios con los grandes supermercados y/o tiendas Hard Discount con el propósito de mantenerse en el mercado aunque esto implique disminuir el margen de utilidad o comprar en grandes cantidades para aprovechar descuentos por parte de las empresas proveedoras; de esta manera es difícil que la economía se estabilice cuando los pequeños negocios están disminuyendo las utilidades y los almacenes de descuento duro están atrayendo más clientes y expandiendo su tiendas en más puntos y barrios de las ciudades.

Mientras que en esta investigación se evidencia que las tiendas de barrio en una escala de 1 a 10 han sido afectadas en 8 puntos por falta de conocimiento y aplicación de estrategias de planeación en áreas de mercadeo, manejo de inventarios, contabilidad, entre otros. A manera de complemento en el estudio de Sánchez (2018) propone que este sin importar el tamaña del establecimiento comercial se pueden ofrecer y llevar a cabo estrategias de marketing para la mejora del servicio, lo que podría hacerle frente a las HD, debido a que por cada espacio de la torta de mercado que pierden las mimimercados y tiendas de barrio lo ganan las tiendas de descuento duro. Consecuentemente con lo anterior, Rodríguez, Morris, Reyes \& Lorena, (2019:49) concluyeron que "las tiendas de barrio, siguen siendo la principal vitrina para los productos de consumo masivo, es el desvare y para el cliente es muy importante el fiado, y el que puede comprar en pequeñas 
cantidades en las tiendas de barrio". Frente a este argumento los resultados de éste estudio coinciden al evidenciar que los propietarios fundamentan su desarrollo basados en la vivencia del día a día y el conocimiento de su clientela haciendo de esta relación un proceso más personalizado y fidelizado por las ventas al por menor.

Igualmente, Fernández \& Carrillo (2017) en su investigación de HD logran concluir que la mayor parte de su clientela continúa con ellos pero que igualmente deben mejorar sus establecimientos en beneficio de la protección de su mercado, mientras tanto, el presente estudio arrojo como resultado que la disminución en las ventas se origina en la falta de estrategias de mercado y en el modelo de administración, es decir, que la disminución de clientes e ingresos es notoria y ha causado impactos negativos en la economía de todo el país.

\section{Conclusiones}

La presente investigación permitió concluir que las tiendas HD han venido afectando paulatinamente el crecimiento económico de los minimercados existentes en las tres principales ciudades del departamento de Boyacá, adicionalmente a esto, se establece que existe un bajo nivel de formación en mercadeo de los propietarios, lo que conlleva a que la inexistencia de procesos administrativos acordes a las microempresas objeto de estudio.

De la misma manera, se logró establecer que los minimercados llevan un buen tiempo de existencia en el mercado y han logrado sobrevivir basados en el menudeo y en el proceso de venta cara a cara con fundamento en la amistad y el conocimiento de sus clientes, denotando una baja en el número de clientes habituales del establecimiento por la llegada de los almacenes de descuento duro.

Los factores que han afectado a los tenderos tienen que ver directamente con la comercialización de los productos de la canasta familiar a un bajo precio, específicamente las tiendas de descuento duro con las promociones que son del 10,20 y $30 \%$ en toda la tienda, a tal punto que los negocios han observado la disminución de clientes e indican cada uno ha dejado de comprar en promedio entre 1 USD y 3 USD diarios.

Por último, la investigación permitió concluir que la mayoría de los encuestados No tienen estrategias de marketing, siendo los precios, la publicidad y las promociones las estrategias más ausentes en dichos establecimientos, dicho fenómeno se puede asociar a la escolaridad de los tenderos puesto que sus conocimientos en mercadeo y publicidad son mínimas.

Es de reiterar que la política de precios es la más agresiva por su impacto psicológico en los estratos más bajos, y aunque los propietarios de los minimercados traten de igualar los precios sigue siendo esta la variable que más ha venido reduciendo los clientes. De otro lado, el factor que afecta en menor medida es la amplitud de horarios teniendo en cuenta que normalmente las tiendas de barrio están abiertas aproximadamente desde las 6.30 am hasta las 8 pm en jornada continua.

\section{Referencias Bibliográficas}

Almeyda, J. D. (2016). El fenómeno de las tiendas de descuento en el mercado del retail colombiano. Recuperado de: http://hdl.handle. net/10654/15802. 
Asociación Nacional De IndustrialesANDI. (2017) Colombia: Balance 2016 y Perspectivas 2017, 44. Recuperado de: http://www.andi.com. co/Documents/Documentos $\% 20$ 2016/ANDI-Balance\%202016Perspectivas2017.pdf

Beltrán, A. R. (2016). Cadenas de descuento duro como competencia a las tiendas de barrio de Bogotá. Universidad Militar Nueva Granada, Bogotá. D.C. Recuperado de: http:// hdl.handle.net/10654/15575.

Bossa Gracia, G. A. (2012). Impacto del sistema de venta al menudeo en el comportamiento de compra de las marcas comercializadas en las tiendas de barrio de la ciudad de Cartagena. Saber, Ciencia y Libertas, 7(2), 77-98.

Caicedo, J.M., \& Quiceno, J.M. (2015). Situación Actual de la Tienda de Barrio Frente a la Aparición de las Nuevas Superficies ARA y D1 en la Ciudad de Manizales. Recuperdo de http://ridum.umanizales. edu.co:8080/xmlui/bitstream/handle $/ 6789 / 2080 /$ trabajo $\% 20$ de $\% 20$ grado.pdf?sequence $=1$

Camacho, Briceida (2003), Metodología de la Investigación científica, un camino fácil de recorrer para todos. Tunja, Colombia. Editorial UPTC.

Cámara de Comercio Tunja, (2017). Boyacá en cifras 2015-2016 Recuperado de: http://ccomerciotunja.org. co/ccomercio/boyacaencifras/BoyacaEnCifras2015-2016.pdf

Cliquet, G., \& Guillo, P. A. (2013). Retail network spatial expansion: An application of the percolation theory to hard discounters. Journal of Retailing and Consumer Services. 20(2), 173-181. DOI:https://doi. org/10.1016/j.jretconser.2012.11.005
Dinero, (2016). El fenómeno D1: La revolución de las tiendas de descuento. Dinero, 1-11.

EI Tiempo. (2005). CARULLA: MIS PRIMEROS 100 AÑOS, Diario El tiempo, Febrero.

Fernández Molano, J., \& Carrillo Orjuela, J. E. (2017). Análisis del impacto del ingreso de los establecimientos "hard discount" frente a las tiendas de barrio de la ciudad de Ibagué. Vía Innova, (4), 47 - 56. https://doi. org/10.23850/2422068X.1180

Gijsbrechts, E., Campo, K. \& Vroegrijk, M. (2018). Save or (over-)spend? The impact of hard-discounter shopping on consumers' grocery outlay. International Journal of Research in Marketing, DOI: https://doi. org/10.1016/j.ijresmar.2018.01.004

Gómez, A., Jiménez, J. y Perdiguero, J. (2015). La entrada de un supermercado de descuento duro: efecto sobre precios. Recuperado de: http://repositorio.uchile.cl/handle/2250/141213

González, J. (2009). El capital intelectual y sus indicadores en el sector industrial. Revista TEACS, 1(2), 9-33. Recuperado de http://www.ucla. edu.ve/dac/revistateacs/articulos/ Rev2-Art1-Gonzalez.pdf

González, J., Cerón, C., Alcázar, F. (2010). Caracterización Emprendedora De Los Empresarios En Los Valles De Tundama $Y$ Sugamuxi, Boyacá. Pensamiento y Gestión, Universidad del Norte, 29 (Julio), 163-189

González, J., Rodríguez, M. \& Moreno, L. (2014). Caracterización de la gestión estratégica de las grandes empresas del Valle de Sugamuxi del departamento de Boyacá, Colombia. Entramado, Enero-Junio, 106-124. 
Hernández Fernández, L., Portillo Medina, R., Romero Borré, J., \& Hernández Chacín, A. (2015). Gobierno corporativo y profesionalización empresarial en hipermercados y supermercados familiares en Venezuela. Utopia y Praxis Latinoamericana, 20(68), 116-135.

Jaramillo, A. P., Neita Pérez, J. P., Pirazán Pirazán, S. P. \& Villarraga Torres, C. D. (2017). Análisis de caso de estrategias financieras de las tiendas de descuento Duro D1, Justo y Bueno y Ara en Colombia. Trabajo de Grado. Universidad Católica de Colombia. Facultad de Ciencias Económicas y Administrativas. Programa de Economía. Especialización en Administración Financiera. Bogotá, Colombia

López, D. \& Peñalosa M. (2019). Responsabilidad social empresarial y su impacto en la disposición a pagar más entre los consumidores colombianos. Revista Venezolana de Gerencia, 24(87), 654-667. DOI: http:// dx.doi.org/10.31876/revista.v24i87

Lourenço, C. J. S., \& Gijsbrechts, E. (2013). The impact of national brand introductions on hard-discounter image and share-of-wallet. International Journal of Research in Marketing, 30(4), 368-382. DOI: https://doi. org/10.1016/j.jiresmar.2013.04.005

Martín Cerdeño, V. (2001). Los establecimientos de descuento en el entorno distributivo actual. Esic Market, 110, 169-182.

Martínez, M. P., Silveira Dias, K. T., Braga Junior, S. S., \& da Silva, D. (2017). La logística inversa como herramienta para la gestión de residuos de los supermercados de venta al por menor. Revista De Gestão Ambiental E Sustentabilidade (Geas), 6(3), 150-165. DOI: doi:10.5585/geas. v6i3.519
Méndez, C. (2006). Metodología, Guía para Elaborar Diseños de investigación en Ciencias Administrativas, Económicas y Contables. Editorial Mc.Graw-Hill,.2da edición, México, 03-1999 / 170pp

Morales, D. P. (2012). Tiendas de barrio en Colombia. (Spanish). Pensamiento \& Gestión, 3. Recuperado de: http://search.ebscohost. com/login.aspx?direct $=$ true $\& d b=$ fua\&AN $=82498143 \&$ lang $=e s \&$ site $=e-$ host-live

Plazas, E. R. (2008). ¿Por Qué Las Tiendas De Barrio En Colombia No Fracasaron Frente a la llegada de las Grandes Cadenas de Supermercados?. Entornos, 1(21), 37-50. Recuperado de: https://www.journalusco.edu.co/index.php/entornos/article/view/389/722\%0Ahttp:// blogs.uninorte.edu.co/dparamo. php/2009/03/19/ipor-que-las-tiendas-de-barrio-en-colomb

Prada Ospina, R., \& Ocampo Vélez, P. C. (2016). Oportunidad para adoptar estrategias de orientación al mercado y la orientación a la gerencia de la cadena de abastecimiento en el sector de retail: un caso de estudio. Lámpsakos, (16), 75-87. DOI: doi:10.21501/21454086.2028

Rodríguez, D. C., Morris Diaz, D. M., Reyes, J., \& Lorena, A. (2019). Estrategias gerenciales aplicadas a las tiendas de barrio con el fin de minimizar el impacto que han tenido con la llegada al mercado de las tiendas D1, Justo \& Bueno y Ara. Tesis Doctoral, Corporación Universitaria Minuto de Dios, Bogotá, Colombia.

Salhin, A. F. (2013). The impact of hard discount control mechanism on the discount volatility of UK closed-end funds. Investment Management and Financial Innovations, 10(3), 68-75. 
Sampieri, Roberto, Fernández, Carlos, Baptista, Pilar (2010), Metodología de la investigación, México D.F., Mc Graw hill

Sánchez Duarte, L. A. (2018). El impacto de los establecimientos Hard Discount o tiendas de descuento en el sector comercial de Colombia. Retrieved from https://ciencia.lasalle.edu.co/administracion_de_empresas/1593

Schwalb, M. \& García, M. (2019). Escala de medición de la responsabilidad social del marketing. Revista Venezolana de Gerencia, 24 (87), 701-724. DOI: http://dx.doi. org/10.31876/revista.v24i88

Semana. (2017). La revolución de las tiendas de descuento. Semana. Com, 2-5.

Tamayo, Mario (2002), EI Proceso de la Investigación Científica, La Inves- tigación Científica. Balderas, Mexico. Limusa Noriega Editores

Torres Gastelú, C. A. (2012). La participación de las grandes cadenas de supermercados en las redes de comercialización de los pequeños productores. Nueva Antropología: Revista De Ciencias Sociales, 25(77), 109-132.

Vroegrijk, M., Gijsbrechts, E., \& Campo, K. (2016). Battling for the Household's Category Buck: Can Economy Private Labels Defend Supermarkets Against the Hard-Discounter Threat?. Journal of Retailing, 92(3), 300318. DOI: https://doi.org/10.1016/j. jretai.2016.05.003

Zipitría, L. (2011). Impacto económico del Supermercadismo. Revista de Ciencias Empresariales y Economía, Montevideo, (10), 73-86.

- Esta obra está bajo una licencia de Creative Commons Reconocimiento-NoComercialCompartirlgual 3.0 Unported. http://creativecommons.org/licenses/by-nc-sa/3.0/deed.es_ES 